\title{
Auricular Chondritis in a Postpartum Flare of SLE and APS
}

\section{Elodie Ponce ${ }^{a}$, Bertrand Lioger ${ }^{a, b}$, Yves Gruel $^{c}$, Jerome Potin ${ }^{d}$, Francois Maillot $^{a}$}

\author{
${ }^{a}$ Department of Internal Medicine, Bretonneau Hospital, CHRU, Tours, France \\ ${ }^{\mathrm{b}}$ François Rabelais University, Tours, France \\ ${ }^{c}$ Department of Haematology-Haemostasis, Trousseau Hospital, CHRU, Tours, France \\ ${ }^{d}$ Department of Obstetrics and Gynecology, Bretonneau Hospital, CHRU, Tours, France
}

\begin{abstract}
Introduction: Auricular chondritis has been occasionally described in patients with systemic lupus erythematosus (SLE) and antiphospholipid syndrome (APS).

Materials and methods: We report the case of a woman with a previous history of APS who presented with auricular chondritis with onset of SLE symptoms during the postpartum period.
\end{abstract}

Conclusion: SLE and APS should be taken into consideration in the differential diagnosis of auricular chondritis.

Keywords: Auricular chondritis, systemic lupus erythematosus, antiphospholipid syndrome, post partum

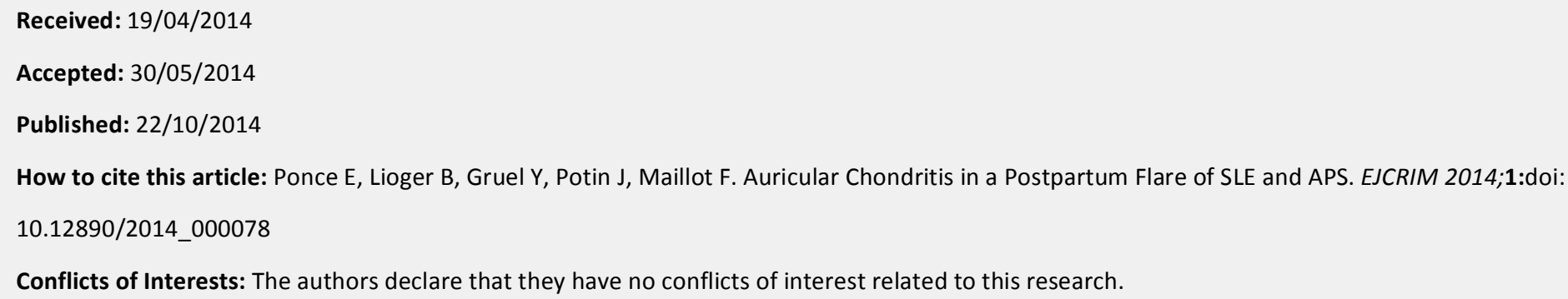

Conflicts of Interests: The authors declare that they have no conflicts of interest related to this research.

\section{Introduction}

Rheumatological manifestations of systemic lupus erythematosus (SLE) are present in $80 \%$ of SLE patients. Arthralgias are mostly bilateral, symmetric and non-erosive, affecting the small joints of the hands and feet. However, there is less information about the involvement of cartilaginous tissue, previously described by Kitridou et al. (1). Thus, chondritis, characterized by inflammation of cartilage, is usually described as relapsing chondritis or granulomatosis with polyangiitis. We report a case of migratory auricular chondritis during a postpartum flare of SLE and antiphospholipid syndrome (APS). 


\section{Case report}

A healthy 44-year-old man presented with recurrent episodes of spontaneous and intermittent skin bleeding. He bled especially at night from different parts of his body, especially the face, forearms, trunk and limbs, but not from his mucous membranes. The episodes were limited to 1-2 min, and there was no standard time for recurrence.

His medical history showed no underlying illness and he took no medication or other toxins. He had lived in Portugal for several years and had no pets or exposure to other animals. There was no history of physical trauma, but his family was experiencing an extremely stressful time and his mother had died recently. No one else in his family had every suffered from a similar condition.

Physical examination showed excretion of bloody sweat (Fig. 1). The colour of tears, saliva and urine was normal.

The results of extensive laboratory tests were normal, including complete blood count and coagulation tests (erythrocyte count $5.77 \times 10^{12} / \mathrm{l}$; haemoglobin: $16.1 \mathrm{~g} / \mathrm{l}$; haematocrit: $44 \%$; mean cell volume (MCV) $86 \mathrm{fl}$; platelets: $330 \times 10^{9}$ /l; Activated Partial Thromboplastin Time (APTT): $22.8 \mathrm{~s}$; prothrombin time: $11.9 \mathrm{~s}$ ). The bloody sweat from his forehead was positive to the benzidine test and microscopic examination of the excreted fluid revealed red blood cells and other components of peripheral blood. The patient refused to have a skin biopsy performed. He was started with alprazolam $0.25 \mathrm{mg}$ once daily and the episodes started to decrease after 2 weeks.

A 30-year-old Caucasian woman, with a history of atopy and allergy to aspirin, was diagnosed in 2008 with APS following a left sural thrombosis together with persistent anticardiolipin antibodies $(\mathrm{aCL})$ and a lupus anticoagulant. Laboratory tests showed a positive Coombs test, as well as antinuclear and anti-DNA antibodies. The patient had never shown any signs of SLE or other systemic autoimmune disease at that time. She received chronic warfarin treatment. Information about the need for an in-hospital aspirin tolerance test before any pregnancy was given to the patient. In 2010, warfarin was changed to enoxaparin on an emergency basis because of an unplanned pregnancy. Aspirin therapy had been considered but not prescribed in view of her medical history and because the aspirin tolerance test was not performed before the patient became pregnant.

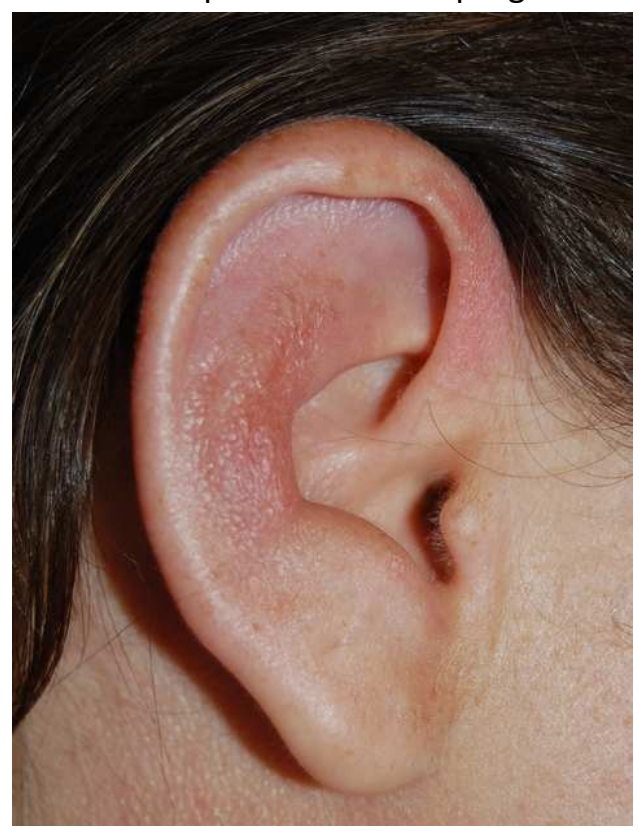

At 25 weeks of amenorrhea, she was admitted for miscarriage due to intra-uterine fetal death. She was discharged 2 days after delivery.

One week later, she was admitted again because she had bilateral hand and wrist arthritis, fever, cutaneous lesions and right auricular swelling. Her body temperature was $38.2^{\circ} \mathrm{C}$. The right ear was moderately swollen, erythematous, warm and tender (Fig. 1)

No nasal or tracheal cartilage changes were observed, but the left ear became involved a few days later. Skin changes were limited to cutaneous necrotic and purpuric lesions of the neck and some fingers, as well as livedo reticularis of the lower limbs. No mucosal lesions were observed. The wrists, hands and fingers were swollen and tender. Cardiac, digestive, pulmonary and neurological examinations were normal. SLE was considered a potential cause of these symptoms and the SLEDAI score was evaluated at 20.

Figure 1: Auricular chondritis of the right ear with inflammation of the cartilage of the pinna, 
Laboratory tests showed plasma haemoglobin $105 \mathrm{~g} / \mathrm{L}, \mathrm{CRP} 39 \mathrm{mg} / \mathrm{L}$ and fibrinogen $5.65 \mathrm{~g} / \mathrm{L}$. TCA was spontaneously at 2.35, due to lupus anticoagulant. Serum creatinine was $74 \mu \mathrm{mol} / \mathrm{L}$ and proteinuria $0.66 \mathrm{~g} /$ day, then $1 \mathrm{~g} /$ day. Antinuclear antibodies were positive at 1:1600 without any specific pattern and anti-DNA antibodies were positive at $82 \mathrm{IU} / \mathrm{mL}$ (normal $<15 \mathrm{IU} / \mathrm{mL}$ by ELISA). ANCA and cryoglobulinaemia were negative. Tests for anti-cartilage antibodies (anti-type 2 collagen autoantibody by ELISA) were negative. C4 was $0.13 \mathrm{~g} / \mathrm{L}(0.14-0.33)$ and C3 was $1.06 \mathrm{~g} / \mathrm{L}(0.69-1.32)$. Skin biopsy of the neck showed signs of thrombotic vasculopathy with fibrin-rich microthrombi in small dermal vessels. A left percutaneous renal biopsy showed features of mesangial lupus nephritis Class II (WHO) without endocapillary or extracapillary proliferative glomerulonephritis. ECG, echocardiography, chest and hand x-rays were normal. The patient was treated with intravenous methylprednisolone $240 \mathrm{mg} /$ day for 3 days and subsequently with prednisone 1 $\mathrm{mg} / \mathrm{kg} /$ day. Enoxaparin was changed to warfarin, and hydrochloroquinine (400 mg/day) was also prescribed. All symptoms resolved within a few days. Renal outcome was favourable. An intravenous aspirin test was also performed and showed signs of intolerance. In September 2011, the patient experienced a new and planned pregnancy. She was then treated with aspirin (100 mg/day), enoxaparin $(0.6 \mathrm{ml} / 12 \mathrm{~h})$, hydroxychloroquine $(400 \mathrm{mg} /$ day) and prednisone $(5$ $\mathrm{mg} /$ day) with a favourable outcome for both the pregnancy and the postpartum period.

\section{Discussion}

In the case reported here, we have described the postpartum onset of SLE symptoms in the course of APS. SLE manifestations have been estimated to occur in 3-8\% of patients previously diagnosed with definite APS (2). These data suggest that most patients with APS do not experience SLE onset over time. In our case, simultaneous SLE and APS flares were linked to the postpartum period. In fact, the postpartum period has been definitely recognized as a risk factor for SLE- and APS-related events. Moreover, a previous positive Coombs test has been shown to be associated with a significant risk of developing SLE in the course of APS (2). As we were aware of such risks, our patient has been informed to contact our team promptly in case of any new symptom following delivery, in order that specific therapy can be provided without delay. Although so far there is no evidence to support this hypothesis, administration of hydroxychloroquine for the first pregnancy might have reduced the risk of SLE onset in the course in this patient. Therefore, the second pregnancy was planned.

Among SLE symptoms in this case, auricular chondritis is noteworthy as it is a rare manifestation of SLE or APS. Indeed, in a study including 400 patients, Kitridou et al. described auricular chondritis only in three patients (1), suggesting that the incidence of auricular chondritis is very low $(<1 \%)$, although these data were not confirmed by other large studies as most cases have been published as single case reports (3). In APS, auricular chondritis appears to be even less frequent as compared with SLE (4). Association of relapsing polychondritis (RP) with SLE and with APS has been described (5). However, in our case, a diagnosis of RP was ruled out in view of the clinical findings and the patient's outcome. Auricular chondritis might have been related to SLE, APS or both conditions in our patient as she presented with chondritis and symptoms of SLE and APS at the same time. However, such a supposition is irrelevant as regards therapeutic options, as corticosteroids are the first-line treatment for chondritis in case of either SLE or APS. 


\section{European Journal}

of Case Reports in

Internal Medicine

\section{Learning Points}

- Apart from relapsing polychondritis, chondritis may be a feature of systemic lupus erythematosus (SLE) and antiphospholipid syndrome (APS).

- The postpartum period is high risk for autoimmune disease flares.

- It is rare for patients previously diagnosed with definite APS to also have SLE.

\section{References}

1. Kitridou RC, Wittmann AL, Quismorio FP Jr. Chondritis in systemic lupus erythematosus: clinical and immunopathologic studies. Clin Exp Rheumatol 1987;5:349-353.

2. Gómez-Puerta JA, Martín H, Amigo M-C, Aguirre MA, Camps MT, Cuadrado MJ, et al. Long-term follow-up in 128 patients with primary antiphospholipid syndrome: do they develop lupus? Medicine (Baltimore) 2005;84:225230.

3. Bellon N, Anguel N, Vandendries C, Goujard C, Lambotte O. Auricular chondritis and thrombotic microangiopathy: an unusual combination revealing systemic lupus erythematosus. Joint Bone Spine 2013;80:424-425.

4. Sciascia S, Bazzan M, Baldovino S, Vaccarino A, Rossi D, Russo A, et al. Antiphospholipid syndrome and relapsing polychondritis: an unusual association. Lupus 2011;20:1336-1337.

5. Zeuner M, Straub RH, Rauh G, Albert ED, Schölmerich J, Lang B. Relapsing polychondritis: clinical and immunogenetic analysis of 62 patients. J Rheumatol 1997;24:96-101. 